\title{
Tailsitter Heading Estimation Using a Magnetometer
}

Timothy McLain

Mechanical Engineering Department, Brigham Young University, mclain@byu.edu

Jason M. Beach

Mechanical Engineering Department, Brigham Young University

Matthew E. Argyle

Department of Electrical Engineering, Brigham Young Univeristy

Randall W. Beard

Department of Electrical Engineering, Brigham Young University

Stephen Morris

MLB Company

Follow this and additional works at: https://scholarsarchive.byu.edu/facpub

Part of the Mechanical Engineering Commons

\section{Original Publication Citation}

Beach, J., Argyle, M., McLain, T., Beard, R., and Morris, S. Tailsitter Heading Estimation Using a Magnetometer, Proceedings of the American Control Conference, pp. 91-96, June 2014, Portland, Oregon.

\section{BYU ScholarsArchive Citation}

McLain, Timothy; Beach, Jason M.; Argyle, Matthew E.; Beard, Randall W.; and Morris, Stephen, "Tailsitter Heading Estimation Using a Magnetometer" (2014). Faculty Publications. 1897.

https://scholarsarchive.byu.edu/facpub/1897 


\title{
Tailsitter Heading Estimation Using a Magnetometer
}

\author{
Jason M. Beach, Matthew E. Argyle, Timothy W. McLain, \\ Randal W. Beard and Stephen Morris
}

\begin{abstract}
The tailsitter aircraft merges the endurance and speed of fixed-wing aircraft with the flexibility and VTOL abilities of rotorcraft. Typical control and estimation schemes make assumptions about the maximum attitude an aircraft will experience that are not valid for tailsitters. This paper discusses the limitations of a typical EKF magnetometer measurement update that uses Euler angles. It is shown how to use a second set of Euler angles to avoid gimbal lock. A method is given that bypasses the use of Euler angles altogether and directly uses the quaternion to determine heading error and update the attitude estimate. This method highlights the EKF limitations in estimating a quaternion. A multiplicative EKF is briefly explored to overcome these limitations. Hardware results on an actual tailsitter aircraft are presented.
\end{abstract}

\section{INTRODUCTION}

The tailsitter aircraft design adds vertical takeoff and landing (VTOL) capabilities to a fixed-wing aircraft without the mechanical complexity of tilt-rotors or typical thrust vectoring. A few manned tailsitters were attempted in the early 1950 s, but were abandoned because of piloting difficulties. As autopilot technology has advanced, the tailsitter concept has become a viable option to give VTOL capabilities to a fixed-wing unmanned aircraft. Brigham Young University is partnering with MLB Company to develop the flight controls of the MLB developed Vertical Bat (V-Bat) aircraft [1], [2]. The current V-Bat prototype is shown in Figure 1. To date, the V-Bat has flown successfully several times, but only with a simple rate-damping stabilization system and a remote control pilot. Autonomous control algorithms for all flight regimes have been designed and implemented in simulation. Implementation in hardware is underway.

A tailsitter aircraft naturally requires an estimator that is functional at all attitudes. In addition to choosing an attitude representation that is free of singularities, measurement updates must also be singularity free. The typical way of incorporating a magnetometer measurement uses Euler angles [3] and breaks down in hover because of gimbal lock. In [4], Stone indicates a magnetometer is used to determine heading on the T-Wing tailsitter aircraft, but nothing specific is given on how the measurement is incorporated into the attitude estimate.

Jason Beach is a graduate student with the Dept. of Mech. Eng., Brigham Young University, Provo, Utah, 84602 jason.m. beach@gmail.com

Matthew Argyle is a PhD candidate with the Dept. of Elec. Eng., Brigham

Young University, Provo, Utah, 84602 matt.argyle@gmail.com

Tim McLain is with the Department of Mechanical Eng., Brigham Young University, Provo, Utah, 84602 mclain@byu.edu

Randy Beard is with the Department of Electrical Eng., Brigham Young University, Provo, Utah, 84602 beard@byu.edu

Stephen Morris is president of the MLB Company smorris@spyplanes.com

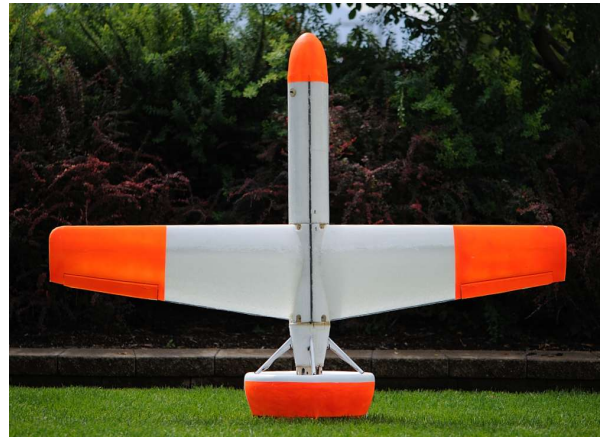

Fig. 1: V-Bat Prototype. This prototype has a six foot wing span and is six feet tall.

This paper presents methods for incorporating magnetometer measurements that work at all attitudes. The typical Extended Kalman Filter (EKF) magnetometer measurement update and methods for overcoming its limitations around gimbal lock are discussed. A Multiplicative Extended Kalman Filter (MEKF) that overcomes the limitations of quaternion estimation with an EKF is also discussed. Preliminary simulation and flight test results are also presented.

\section{Attitude Representation}

At the most fundamental level, attitude representation consists of expressing the relationship between different coordinate frames. With regard to UAVs, three coordinate frames are of particular interest: the inertial coordinate frame which is fixed at an arbitrary location, the vehicle frame [3], and the body frame. The vehicle frame is a copy of the inertial frame that is translated to the vehicle center of mass. Both the inertial and vehicle frames are oriented such that the $x$-axis points north, $y$-axis points east and $z$-axis points to the center of the earth. The body-fixed frame follows standard convention with the $x$-axis pointing out of the tailsitter nose, the $y$-axis out the right wing and the $z$-axis out the bottom of the fuselage.

\section{A. Level Flight Euler Angles}

Euler angles represent attitude as three consecutive rotations about body-fixed axes with the 3-2-1 rotation sequence being the most common in aircraft literature. To perform the overall rotation several intermediate coordinate system definitions are needed. They are [5]:

1) Rotate the vehicle frame about the $\mathbf{k}^{v}$ axis by $\psi_{\ell}$ into the vehicle- 1 frame. 
2) Rotate the vehicle- 1 frame about the $\mathbf{j}^{v 1}$ axis by $\theta_{\ell}$ into the vehicle- 2 frame.

3) Rotate the vehicle- 2 frame about the $\mathbf{i}^{v 2}$ axis by $\phi_{\ell}$ into the body frame.

With sine and cosine abbreviated as "s" and "c," respectively, these rotations mathematically are

$$
\begin{gathered}
\mathbf{R}_{v 2}^{b}\left(\phi_{\ell}\right) \triangleq\left[\begin{array}{ccc}
1 & 0 & 0 \\
0 & \mathrm{c}_{\phi_{\ell}} & \mathrm{s}_{\phi_{\ell}} \\
0 & -\mathrm{s}_{\phi_{\ell}} & \mathrm{c}_{\phi_{\ell}}
\end{array}\right], \quad \mathbf{R}_{v 1}^{v 2}\left(\theta_{\ell}\right) \triangleq\left[\begin{array}{ccc}
\mathrm{c}_{\theta_{\ell}} & 0 & -\mathrm{s}_{\theta_{\ell}} \\
0 & 1 & 0 \\
\mathrm{~s}_{\theta_{\ell}} & 0 & \mathrm{c}_{\theta_{\ell}}
\end{array}\right], \\
\mathbf{R}_{v}^{v 1}\left(\psi_{\ell}\right) \triangleq\left[\begin{array}{ccc}
\mathrm{c}_{\psi_{\ell}} & \mathrm{s}_{\psi_{\ell}} & 0 \\
-\mathrm{s}_{\psi_{\ell}} & \mathrm{c}_{\psi_{\ell}} & 0 \\
0 & 0 & 1
\end{array}\right], \\
\mathbf{R}_{v}^{b}\left(\phi_{\ell}, \theta_{\ell}, \psi_{\ell}\right)=\mathbf{R}_{v 2}^{b}\left(\phi_{\ell}\right) \mathbf{R}_{v 1}^{v 2}\left(\theta_{\ell}\right) \mathbf{R}_{v}^{v 1}\left(\psi_{\ell}\right) .
\end{gathered}
$$

$\mathbf{R}_{v}^{b}\left(\phi_{\ell}, \theta_{\ell}, \psi_{\ell}\right)$ is the Euler angle based rotation matrix representing the rotation of the vehicle frame to the body frame. The subscript on $\mathbf{R}$ indicates the coordinate frame being rotated and the superscript indicates the frame being rotated to. The subscript on the angles distinguishes between the standard level flight Euler angles and the hover Euler angles described in the following section.

\section{B. Hover Euler Angles}

It is well known that for the level flight Euler angle rotation sequence, when $\theta_{\ell}=90^{\circ}$, one degree of freedom is lost in a condition called gimbal lock. In this state, $\phi_{\ell}$ and $\psi_{\ell}$ are ambiguous. To give physical intuition when the tailsitter is in hover, a new set of Euler angles similar to that given in [4] is developed.

The hover coordinate frame is located at the tailsitter center of mass with the $x$-axis pointing up, the $y$-axis pointing east and the $z$-axis pointing north. Note that this is simply the vehicle frame rotated by $90^{\circ}$ about the $\mathbf{j}^{v}$ axis. The complete 1-2-3 rotation sequence is:

1) Rotate the vehicle frame $90^{\circ}$ about the $\mathbf{j}^{v}$ axis into the hover frame.

2) Rotate the hover frame about the negative $\mathbf{i}^{h}$ axis by $\phi_{h}$ into the hover-1 frame. Rotating about the negative $\mathbf{i}^{h}$ axis is necessary to maintain heading sense (i.e., so that $\phi_{h}=90^{\circ}$ still results in the belly pointing east).

3) Rotate the hover- 1 frame about the $\mathbf{j}^{h 1}$ axis by $\theta_{h}$ into the hover- 2 frame.

4) Rotate the hover- 2 frame about the $\mathbf{k}^{v 2}$ axis by $\psi_{h}$ into the body frame.

Mathematically this is represented by

$$
\begin{array}{cc}
\mathbf{R}_{h 2}^{b}\left(\psi_{h}\right) \triangleq\left[\begin{array}{ccc}
\mathrm{c}_{\psi_{h}} & \mathrm{~s}_{\psi_{h}} & 0 \\
-\mathrm{s}_{\psi_{h}} & \mathrm{c}_{\psi_{h}} & 0 \\
0 & 0 & 1
\end{array}\right], & \mathbf{R}_{h 1}^{h 2}\left(\theta_{h}\right) \triangleq\left[\begin{array}{ccc}
\mathrm{c}_{\theta_{h}} & 0 & -\mathrm{s}_{\theta_{h}} \\
0 & 1 & 0 \\
\mathrm{~s}_{\theta_{h}} & 0 & \mathrm{c}_{\theta_{h}}
\end{array}\right], \\
\mathbf{R}_{h}^{h 1}\left(-\phi_{h}\right) \triangleq\left[\begin{array}{ccc}
1 & 0 & 0 \\
0 & \mathrm{c}_{\phi_{h}} & -\mathrm{s}_{\phi_{h}} \\
0 & \mathrm{~s}_{\phi_{h}} & \mathrm{c}_{\phi_{h}}
\end{array}\right], & \mathbf{R}_{v}^{h} \triangleq\left[\begin{array}{ccc}
0 & 0 & -1 \\
0 & 1 & 0 \\
1 & 0 & 0
\end{array}\right], \\
\mathbf{R}_{v}^{b}\left(\phi_{h}, \theta_{h}, \psi_{h}\right)=\mathbf{R}_{h 2}^{b}\left(\psi_{h}\right) \mathbf{R}_{h 1}^{h 2}\left(\theta_{h}\right) \mathbf{R}_{h}^{h 1}\left(-\phi_{h}\right) \mathbf{R}_{v}^{h} .
\end{array}
$$

Like any other sequence of three rotations, this too is affected by gimbal lock when $\theta_{h}= \pm 90^{\circ}$. To cover a full range of attitudes it is necessary to switch between standard aircraft
Euler angles and these hover Euler angles depending on pitch. Also, it is important to note that in deriving the hover Euler angles, we have chosen to maintain $\phi, \theta$ and $\psi$ as rotations about the respective $x, y$ and $z$ axes. Doing so means that when using the level Euler angles, $\psi_{\ell}$ defines heading, but in hover mode $\phi_{h}$ does.

\section{Quaternions}

Instead of three separate rotations, a quaternion expresses attitude in terms of a single rotation. The Euler-Rodrigues symmetric parameters are related to a unit-length axis of rotation $\mathbf{e}$ and an angle of rotation $\Theta$ by

$$
\eta \triangleq\left[\begin{array}{c}
\eta_{0} \\
\eta
\end{array}\right]=\left[\begin{array}{c}
\cos \frac{\Theta}{2} \\
\mathbf{e} \sin \frac{\Theta}{2}
\end{array}\right]
$$

Here we have chosen to place the scalar element as the first component and to express the vector part in bold. The individual components of the vector are expressed with a subscript $x, y$, and $z$. The rotation matrix based on a quaternion is given by [3]

$$
\begin{aligned}
& \mathbf{R}_{v}^{b}(\eta)= \\
& {\left[\begin{array}{lll}
\eta_{0}^{2}+\eta_{x}^{2}-\eta_{y}^{2}-\eta_{z}^{2} & 2\left(\eta_{x} \eta_{y}+\eta_{z} \eta_{0}\right) & 2\left(\eta_{x} \eta_{z}-\eta_{y} \eta_{0}\right) \\
2\left(\eta_{x} \eta_{y}-\eta_{z} \eta_{0}\right) & \eta_{0}^{2}-\eta_{x}^{2}+\eta_{y}^{2}-\eta_{z}^{2} & 2\left(\eta_{y} \eta_{z}+\eta_{x} \eta_{0}\right) \\
2\left(\eta_{x} \eta_{z}+\eta_{y} \eta_{0}\right) & 2\left(\eta_{y} \eta_{z}-\eta_{x} \eta_{0}\right) & \eta_{0}^{2}-\eta_{x}^{2}-\eta_{y}^{2}+\eta_{z}^{2}
\end{array}\right] .}
\end{aligned}
$$

A quaternion must be unit-norm to represent a rotation. Successive rotations are easily expressed as a single quaternion through the composition operator

$$
\eta^{\prime \prime}=\eta^{\prime} \otimes \eta=\left[\begin{array}{c}
\eta_{0}^{\prime} \eta_{0}-\boldsymbol{\eta}^{\prime} \cdot \boldsymbol{\eta} \\
\eta_{0}^{\prime} \boldsymbol{\eta}+\eta_{0} \boldsymbol{\eta}^{\prime}-\boldsymbol{\eta}^{\prime} \times \boldsymbol{\eta}
\end{array}\right] .
$$

As with most quaternion operations, composition is not commutative. In (5), $\eta^{\prime \prime}$ represents the overall rotation given by $\eta$ followed by $\eta^{\prime}$.

Quaternions have the advantage of being singularity free and are more computationally efficient; however, they are not without their challenges. One challenge is the unit-norm constraint. Since a quaternion that represents a rotation must be unit-norm, the operations of addition and subtraction are not meaningful. Lastly, any rotation can be represented by two quaternions, $\eta$ and $-\eta$. This non-uniqueness is referred to as double coverage. The typical way of dealing with double coverage is to simply keep the scalar element positive. If the scalar element becomes negative, negating all four elements of the quaternion maintains the same rotation, while protecting against double coverage.

A quaternion can be expressed as level flight Euler angles by [5]

$$
\begin{aligned}
\phi_{\ell} & =\operatorname{atan} 2\left[2\left(\eta_{0} \eta_{x}+\eta_{y} \eta_{z}\right), \eta_{0}^{2}-\eta_{x}^{2}-\eta_{y}^{2}+\eta_{z}^{2}\right], \\
\theta_{\ell} & =\sin ^{-1}\left(2\left(\eta_{0} \eta_{y}-\eta_{x} \eta_{z}\right)\right), \\
\psi_{\ell} & =\operatorname{atan} 2\left[2\left(\eta_{0} \eta_{z}+\eta_{x} \eta_{y}\right), \eta_{0}^{2}+\eta_{x}^{2}-\eta_{y}^{2}-\eta_{z}^{2}\right] .
\end{aligned}
$$

The conversion between quaternions and hover Euler angles 
is given by [6]

$$
\begin{aligned}
\phi_{h} & =\operatorname{atan} 2\left[\eta_{y} \eta_{z}-\eta_{0} \eta_{x}, \eta_{0} \eta_{y}+\eta_{x} \eta_{z}\right], \\
\theta_{h} & =\sin ^{-1}\left[-\eta_{0}^{2}+\eta_{x}^{2}+\eta_{y}^{2}-\eta_{z}^{2}\right], \\
\psi_{h} & =\operatorname{atan} 2\left[\eta_{0} \eta_{x}+\eta_{y} \eta_{z}, \eta_{0} \eta_{y}-\eta_{x} \eta_{z}\right] .
\end{aligned}
$$

\section{Attitude Estimation}

The EKF has become a standard tool in attitude estimation since it performs well, is well documented and relatively straight-forward to implement. This section discusses three ways to update a quaternion attitude estimate with a magnetometer measurement:

1) A "pure Euler" method that switches between hover and level Euler angles to determine heading and perform what we will call the compass Kalman update to update the attitude estimate.

2) A "mixed quaternion Euler" method which calculates heading error using quaternions which is then used in the compass Kalman update.

3) A "pure quaternion" method that uses quaternions to determine heading error and update the attitude estimate.

To overcome the limitations in using an EKF with quaternions a Multiplicative EKF is then examined.

\section{A. Pure Euler Angle Magnetometer Update}

As its name implies, a 3-axis magnetometer provides a vector representation of the earth's magnetic field. In level flight the pure Euler angle method is the traditional and most intuitive way to use a magnetometer-as a digital compass that gives an absolute heading measurement. This level flight method is discussed first and then extended to hover flight.

1) Level Flight: The magnetic vector measured by the magnetometer, $\mathbf{b}^{*}$, is by default expressed in the body frame. To get heading this vector must be expressed in the vehicle-1 frame so that heading is the only rotation between magnetic north and $\mathbf{b}^{*}$. Since the quaternion represents attitude as a single rotation it is not possible to use it directly to express $\mathbf{b}^{*}$ in the vehicle-1 frame. Transposing matrices from (1) gives the inverse rotations needed [3]:

$$
\begin{aligned}
\mathbf{b}^{v_{1}} & =\mathbf{R}_{v 2}^{v 1}\left(\theta_{\ell}\right) \mathbf{R}_{b}^{v 2}\left(\phi_{\ell}\right) \mathbf{b}^{*}, \\
& =\left[\begin{array}{ccc}
\mathrm{c}_{\theta_{\ell}} & \mathrm{s}_{\phi_{\ell}} \mathrm{s}_{\theta_{\ell}} & \mathrm{c}_{\phi} \mathrm{s}_{\theta_{\ell}} \\
0 & \mathrm{c}_{\phi_{\ell}} & -\mathrm{s}_{\phi_{\ell}} \\
-\mathrm{s}_{\theta_{\ell}} & \mathrm{s}_{\phi_{\ell}} \mathrm{c}_{\theta_{\ell}} & \mathrm{c}_{\phi} \mathrm{c}_{\theta_{\ell}}
\end{array}\right] \mathbf{b}^{*} .
\end{aligned}
$$

Magnetic heading is then given by

$$
\psi_{m}=-\operatorname{atan} 2\left[b_{y}^{v 1}, b_{x}^{v 1}\right]
$$

If the true magnetic vector expressed in the vehicle frame is known, declination can be determined by

$$
\delta=\operatorname{atan} 2\left[b_{y}^{v}, b_{x}^{v}\right] .
$$

True heading and heading error are then

$$
\begin{aligned}
& \psi=\psi_{m}+\delta, \\
& \tilde{\psi} \triangleq \psi-\psi_{\ell},
\end{aligned}
$$

where $\psi_{\ell}$ is the estimated heading obtained from (8).

The non-linear measurement output equation for level flight comes from (8). Noting that the rows of $\mathbf{R}_{v}^{b}$ represent the body axes expressed in the vehicle frame and using (4), the elements of $\mathbf{R}_{v}^{b}$ can be used to rewrite (8) as

$$
h_{b, \ell}(\hat{\eta}) \triangleq \psi_{\ell}=\operatorname{atan} 2\left[\mathbf{R}_{v}^{b}(1,2), \mathbf{R}_{v}^{b}(1,1)\right] .
$$

The output matrix $\mathbf{C}_{b}$ is formed by taking the Jacobian of (17) giving

$$
\begin{aligned}
& \frac{\partial h_{b, \ell}}{\partial \eta_{0}}=\frac{2 \eta_{z} \mathbf{R}_{v}^{b}(1,1)}{\mathbf{R}_{v}^{b}(1,1)^{2}+\mathbf{R}_{v}^{b}(1,2)^{2}}, \\
& \frac{\partial h_{b, \ell}}{\partial \eta_{x}}=\frac{2 \eta_{y} \mathbf{R}_{v}^{b}(1,1)}{\mathbf{R}_{v}^{b}(1,1)^{2}+\mathbf{R}_{v}^{b}(1,2)^{2}}, \\
& \frac{\partial h_{b, \ell}}{\partial \eta_{y}}=\frac{2 \eta_{x} \mathbf{R}_{v}^{b}(1,1)+4 \eta_{y} \mathbf{R}_{v}^{b}(1,2)}{\mathbf{R}_{v}^{b}(1,1)^{2}+\mathbf{R}_{v}^{b}(1,2)^{2}}, \\
& \frac{\partial h_{b, \ell}}{\partial \eta_{z}}=\frac{2 \eta_{0} \mathbf{R}_{v}^{b}(1,1)+4 \eta_{z} \mathbf{R}_{v}^{b}(1,2)}{\mathbf{R}_{v}^{b}(1,1)^{2}+\mathbf{R}_{v}^{b}(1,2)^{2}}, \\
& \mathbf{C}_{b} \triangleq \frac{\partial h_{b}}{\partial \eta}=\left[\begin{array}{llll}
\frac{\partial h_{b, \ell}}{\partial \eta_{0}} & \frac{\partial h_{b, \ell}}{\partial \eta_{x}} & \frac{\partial h_{b, \ell}}{\partial \eta_{y}} & \frac{\partial h_{b, \ell}}{\partial \eta_{z}}
\end{array}\right] .
\end{aligned}
$$

The compass Kalman update is then performed by

$$
\begin{aligned}
\mathbf{L}_{b} & =\mathbf{P}^{-} \mathbf{C}_{b}^{\top}\left(\mathbf{C}_{b} \mathbf{P}^{-} \mathbf{C}_{b}^{\top}+\mathbf{R}_{b}\right)^{-1}, \\
\hat{\eta}^{+} & =\hat{\eta}^{-}+\mathbf{L}_{b} \tilde{\psi}, \\
\mathbf{P}^{+} & =\left(\mathbf{I}-\mathbf{L}_{b} \mathbf{C}_{b}\right) \mathbf{P}^{-} .
\end{aligned}
$$

As in other cases, after the update occurs the quaternion should be normalized and corrected for double coverage. If not used in conjunction with hover Euler angles, this update has several problems when $\theta_{\ell}$ is near $90^{\circ}$. First, it is impossible to determine $\phi_{\ell}$ accurately for use in (12). Second, small deviations in $\theta_{\ell}$ cause large swings in heading. For example, if $\theta_{\ell}=90^{\circ}$ with zero bank or heading, (17) gives the correct heading of zero. If $\theta_{\ell}$ is increased to $91^{\circ}$, either actually or by measurement noise, $\psi_{\ell}$ will swing to $180^{\circ}$. Additionally, when $\theta_{\ell}=90^{\circ}, \mathbf{R}_{v}^{b}(1,1)=\mathbf{R}_{v}^{b}(1,2)=0$, which is indicative that using the body $x$-axis to define heading in this state is ambiguous. This also causes $\mathbf{C}_{b}$ and $\mathbf{L}_{b}$ to be zero, resulting in the filter always rejecting the measurement input.

2) Hover Flight: It is not possible to define heading in a manner that is valid at all attitudes; a second definition is needed. When the tailsitter is in hover, heading is defined as the direction the body $z$-axis points as a result of a rotation about the vehicle $z$-axis.

Similar to level flight, magnetic heading, $\psi_{m}$, is determined by expressing the magnetometer measurement in to the hover-1 frame by

$$
\mathbf{b}^{h 1}=\mathbf{R}_{h 2}^{h 1}\left(\theta_{h}\right) \mathbf{R}_{b}^{h 2}\left(\psi_{h}\right) \mathbf{b}^{*},
$$




$$
\begin{aligned}
= & =\left[\begin{array}{ccc}
c_{\theta_{h}} c_{\psi_{h}} & -c_{\theta_{h}} s_{\psi_{h}} & s_{\theta_{h}} \\
s_{\psi_{h}} & c_{\psi_{h}} & 0 \\
-s_{\theta_{h}} c_{\psi_{h}} & s_{\theta_{h}} s_{\psi_{h}} & c_{\theta_{h}}
\end{array}\right] \mathbf{b}^{*}, \\
\psi_{m} & =-\operatorname{atan} 2\left[b_{y}^{h 1}, b_{z}^{h 1}\right] .
\end{aligned}
$$

True heading is calculated using (15); however, since $\phi_{h}$ governs heading while hovering, the heading error given by (16) is instead

$$
\tilde{\psi} \triangleq \psi-\phi_{h} .
$$

Since heading is now defined using the $z$-axis, the nonlinear measurement output equation is

$$
h_{b, h}(\hat{\eta}) \triangleq \phi_{h}=\operatorname{atan} 2\left[\mathbf{R}_{v}^{b}(3,2), \mathbf{R}_{v}^{b}(3,1)\right],
$$

making the partial derivatives for the output matrix $\mathbf{C}_{b}$

$$
\begin{aligned}
\frac{\partial h_{b, h}}{\partial \eta_{0}} & =\frac{-2\left(\eta_{x} \mathbf{R}_{v}^{b}(3,1)+\eta_{y} \mathbf{R}_{v}^{b}(3,2)\right)}{\mathbf{R}_{v}^{b}(3,1)^{2}+\mathbf{R}_{v}^{b}(3,2)^{2}}, \\
\frac{\partial h_{b, h}}{\partial \eta_{x}} & =\frac{-2\left(\eta_{0} \mathbf{R}_{v}^{b}(3,1)+\eta_{z} \mathbf{R}_{v}^{b}(3,2)\right)}{\mathbf{R}_{v}^{b}(3,1)^{2}+\mathbf{R}_{v}^{b}(3,2)^{2}}, \\
\frac{\partial h_{b, h}}{\partial \eta_{y}} & =\frac{2\left(\eta_{z} \mathbf{R}_{v}^{b}(3,1)-\eta_{0} \mathbf{R}_{v}^{b}(3,2)\right)}{\mathbf{R}_{v}^{b}(3,1)^{2}+\mathbf{R}_{v}^{b}(3,2)^{2}}, \\
\frac{\partial h_{b, h}}{\partial \eta_{z}} & =\frac{2\left(\eta_{y} \mathbf{R}_{v}^{b}(3,1)-\eta_{x} \mathbf{R}_{v}^{b}(3,2)\right)}{\mathbf{R}_{v}^{b}(3,1)^{2}+\mathbf{R}_{v}^{b}(3,2)^{2}} .
\end{aligned}
$$

The compass Kalman update in (18)-(21) is then used to update the estimate.

Similar to the level flight Euler angles, a singularity occurs when $\theta_{h}=90^{\circ}$. To avoid this the two representations are used together, switching between them at a given point. Chosen somewhat arbitrarily, when $\theta_{\ell} \leq 60^{\circ}$, the level flight Euler angles are used to update the attitude, and above that the hover flight Euler angles are used.

\section{B. Mixed Quaternion Euler Update}

Using the attitude quaternion, heading error, $\tilde{\psi}$, can be calculated directly without using Euler angles, thus eliminating the need to switch representations to avoid singularities. Instead of expressing $\mathbf{b}^{*}$ in the vehicle- 1 frame, $\mathbf{b}^{*}$ is expressed in the vehicle frame using (4) in

$$
\mathbf{b}^{\hat{v}}=\left[\mathbf{R}_{v}^{b}(\hat{\eta})\right]^{\top} \mathbf{b}^{*},
$$

Because the estimated quaternion is used, (26) will actually give the magnetometer measurement expressed in the estimated vehicle frame, denoted by $\hat{v}$. The estimated angle of declination is

$$
\hat{\delta}=\operatorname{atan} 2\left[b_{y}^{\hat{v}}, b_{x}^{\hat{v}}\right] .
$$

Heading error is simply the difference between the true and estimated values of declination or

$$
\tilde{\psi} \triangleq \delta-\hat{\delta} .
$$

This heading error is then used directly in (20) and has the advantages of being computationally faster and not requiring use of a switch to determine heading error. The compass
Kalman update is then used to update the attitude estimate; however, a switch is still required to determine the output matrix $\mathbf{C}_{b}$ when calculating the Kalman gain.

\section{Pure Quaternion Update}

It is possible to avoid Euler angles altogether by transforming the heading error into a quaternion measurement and feeding that into the update. The error expressed as a quaternion is

$$
\eta_{m e} \triangleq\left[\begin{array}{llll}
\cos \frac{\psi_{e}}{2} & 0 & 0 & \sin \frac{\psi_{e}}{2}
\end{array}\right]^{\top} .
$$

To convert this to an attitude measurement, this error must be composed with the current estimate which is then used in a Kalman update. In this case $\mathbf{C}$ is identity reducing the update to

$$
\begin{aligned}
\mathbf{L}_{\mathbf{b}} & =\mathbf{P}^{-}\left(\mathbf{R}_{\mathbf{b}}+\mathbf{P}^{-}\right)^{-1}, \\
\mathbf{y}_{\mathbf{b}} & =\hat{\eta}^{-} \otimes \eta_{m e}, \\
\hat{\eta}^{+} & =\hat{\eta}^{-}+\mathbf{L}_{\mathbf{b}}\left(\mathbf{y}_{\mathbf{b}}-\hat{\eta}^{-}\right), \\
\mathbf{P}^{+} & =\left(\mathbf{I}-\mathbf{L}_{\mathbf{b}}\right) \mathbf{P}^{-} .
\end{aligned}
$$

As before, after $\hat{\eta}$ is updated the quaternion estimate should be normalized. The primary advantage of this method is that it works at all attitudes without a switch. Computationally, calculating heading error is faster, but the Kalman update is slower because of the matrix inversion required. The conceptual problem with using an EKF to estimate a quaternion is illustrated by $\eta_{m e} \neq \mathbf{y}_{\mathbf{b}}-\hat{\eta}^{-}$.

\section{Multiplicative Extended Kalman Filter}

The EKF in general has two conceptual problems when it comes to estimating a quaternion. Already discussed is the issue of the quaternion geometry and the Kalman update. Another issue pointed out by [7] is that because of the quaternion unit norm constraint, the $4 \times 4$ covariance matrix is redundant. The MEKF was designed to address these two issues. The MEKF implemented was developed in [8] with a magnetometer update being used in lieu of a GPS update.

A key concept to the MEKF is expressing attitude error as a product of the error axis of rotation $\mathbf{e}$ and angle of rotation $\Theta$ in $\mathbf{a}_{\mathbf{e}}=\Theta \mathbf{e}$. Providing that $\mathbf{e}$ is unit-norm, all information about attitude error is recovered and expressed as an error quaternion

$$
\delta \eta\left(\mathbf{a}_{\mathbf{e}}\right) \triangleq\left[\begin{array}{c}
\cos \frac{\left|\mathbf{a}_{\mathbf{e}}\right|}{2} \\
\frac{\mathbf{a}_{\mathbf{e}}}{\left|\mathbf{a}_{\mathbf{e}}\right|} \sin \frac{\left|\mathbf{a}_{\mathbf{e}}\right|}{2}
\end{array}\right] .
$$

As the norm of $\mathbf{a}_{\mathbf{e}}$ goes to zero, calculation of the vector becomes numerically unstable. Markley [7] suggests different error representations, all of which have a similar singularity at some error angle. The approach taken here is to assume the error quaternion is $\left[\begin{array}{cccc}1 & 0 & 0 & 0\end{array}\right]^{\top}$ if the norm of $\mathbf{a}_{\mathbf{e}}$ is below some bound.

1) Prediction Step: The primary difference of the MEKF is that the attitude error $\mathbf{a}_{\mathbf{e}}$ is the state vector. An estimate of the attitude quaternion is also maintained, but it is not formally part of the state vector. An important assumption in making this work is that incorporating attitude error into the 
quaternion estimate at the end of each measurement update resets the attitude error to zero. Because of this, the state vector itself does not require integration and is used only to derive the covariance update

$$
\begin{aligned}
\mathbf{F} & \triangleq\left[\begin{array}{ccc}
0 & r & -q \\
-r & 0 & p \\
q & -p & 0
\end{array}\right], \\
\mathbf{P}^{+} & =\mathbf{P}^{-}+T_{s}\left(\mathbf{F} \mathbf{P}^{-}+\mathbf{P}^{-} \mathbf{F}^{\top}+\mathbf{Q}\right),
\end{aligned}
$$

where $p, q$, and $r$ are the body angular rates, $T_{s}$ is the filter sample rate and $\mathbf{Q}$ is the process noise matrix.

Although not formally part of the state equation, the estimated quaternion must be propagated forward in time separately using

$$
\begin{aligned}
\mathbf{A} & \triangleq \frac{1}{2}\left[\begin{array}{cccc}
0 & -p & -q & -r \\
p & 0 & r & -q \\
q & -r & 0 & p \\
r & q & -p & 0
\end{array}\right], \\
\hat{\boldsymbol{\eta}}^{+} & =\hat{\boldsymbol{\eta}}^{-}+T_{s} \mathbf{A} \hat{\boldsymbol{\eta}}^{-} .
\end{aligned}
$$

2) Accelerometer Measurement Update: To get tilt information, the gravity vector $\mathbf{g}^{v}=\left[\begin{array}{lll}0 & 0 & -1\end{array}\right]^{\top}$ is rotated into the body frame and the accelerometer measurement normalized by

$$
\begin{aligned}
& \mathbf{g}^{b} \triangleq \mathbf{R}_{v}^{b}(\hat{\eta}) \mathbf{g}^{v}, \\
& \mathbf{g}_{\mathbf{m}} \triangleq \frac{\mathbf{a}}{|\mathbf{a}|} .
\end{aligned}
$$

The error angle and axis of rotation are then calculated as

$$
\begin{aligned}
& \Theta_{a} \triangleq \cos ^{-1}\left(\mathbf{g}_{\mathbf{m}} \cdot \mathbf{g}^{b}\right), \\
& \mathbf{e}_{\mathbf{a}} \triangleq \frac{\mathbf{g}_{\mathbf{m}} \times \mathbf{g}^{b}}{\left|\mathbf{g}_{\mathbf{m}} \times \mathbf{g}^{b}\right|} .
\end{aligned}
$$

The attitude error vector is then defined as

$$
\mathbf{a}_{\mathbf{e a}}^{-} \triangleq \Theta_{a} \mathbf{e}_{\mathbf{a}} .
$$

Confidence in the accelerometer measurement is reduced by inflating the accelerometer covariance matrix, $\mathbf{R}_{\mathbf{a}}$, when the norm of the measurement is not equal to gravity by

$$
\mathbf{R}_{\mathbf{a}}=\mathbf{R}_{\mathbf{a}}\left[1+k\left|1-\frac{|\mathbf{a}|}{g}\right|\right],
$$

where $k$ is a tunable gain.

The attitude error before the measurement is assumed to be zero and the measurement output matrix, $\mathbf{C}$, is identity, allowing the Kalman gain and the attitude error update equations to reduce to

$$
\begin{aligned}
\mathbf{L}_{\mathbf{a}} & =\mathbf{P}^{-}\left(\mathbf{R}_{\mathbf{a}}+\mathbf{P}^{-}\right)^{-1} \\
\mathbf{a}_{\mathbf{e a}}^{+} & =\mathbf{L}_{\mathbf{a}} \mathbf{a}_{\mathbf{e a}}^{-} \\
\mathbf{P}^{+} & =\left(\mathbf{I}-\mathbf{L}_{\mathbf{a}}\right) \mathbf{P}^{-}
\end{aligned}
$$

The attitude error is then reset to zero by updating the quaternion using composition

$$
\hat{\boldsymbol{\eta}}^{+}=\delta \eta\left(\mathbf{a}_{\mathbf{e a}}^{+}\right) \otimes \hat{\boldsymbol{\eta}}^{-} .
$$

3) Magnetometer Measurement Update: Calculation of heading error from Section III-B ties in well here. Using (28), the attitude error vector is

$$
\mathbf{a}_{\mathbf{e b}}^{-} \triangleq\left[\begin{array}{lll}
0 & 0 & \tilde{\psi}
\end{array}\right]^{\top} .
$$

The attitude error update is similar to that used by the accelerometers:

$$
\begin{aligned}
\mathbf{L}_{\mathbf{b}} & =\mathbf{P}^{-}\left(\mathbf{R}_{\mathbf{b}}+\mathbf{P}^{-}\right)^{-1} \\
\mathbf{a}_{\mathrm{eb}}^{+} & =\mathbf{L}_{\mathbf{b}} \mathbf{a}_{\mathrm{eb}}^{-} .
\end{aligned}
$$

Resetting the error to zero is done by

$$
\hat{\boldsymbol{\eta}}^{+}=\hat{\boldsymbol{\eta}}^{-} \otimes \delta \eta\left(\mathbf{a}_{\mathbf{e b}}^{+}\right) .
$$

The order of composition is reversed to account for the error being expressed in the vehicle frame. The covariance update is similar to (47).

\section{Testing Results}

Initial testing was conducted in a UAV simulator built in MATLAB according to [6]. Each method discussed performed well in simulation and has been implemented in hardware. The source code of a commercially available autopilot was modified to incorporate the algorithms listed.

The goal in testing was to compare the quaternion estimated by the autopilot to one measured by a motion capture system with sub-millimeter accuracy. Tests were conducted outdoors to allow access to the GPS and magnetometer data needed for attitude estimation. For the testing reported here, the tailsitter was hovered approximately three feet off the ground. An attitude control loop was used to keep the vehicle upright; however, for safety the aircraft was loosely tethered to an overhead cable. Several test flights were conducted. In each one, data was logged by the autopilot and the motion capture system. During each test several heading commands were given to the autopilot via the ground control station.

In each plot heading as calculated by (9) is given. Figure 2 shows data where heading was updated using the pure Euler method. A maximum of $16.3^{\circ}$ of heading error was recorded for this flight. This is somewhat larger than expected and may have been caused by electromagnetic interference from the battery cables. Steps have been taken to mitigate this issue.

Figure 3 shows data using the mixed quaternion Euler approach and with $14.3^{\circ}$ max error, performed similarly to the previous method. Of the three EKF based methods tested, this was computationally the fastest.

The final set of data recorded was obtained using the pure quaternion measurement update. It was noted during simulation that the covariance matrix values that worked well for the above methods did not work well here. Good performance was obtained in simulation once these values were refined. The large error from the experimental flight test shown in Figure 4 is mainly attributed to not being able to re-tune the filter while the outdoor motion capture system was available.

The MEKF implemented was not ready for flight testing when the other flights were conducted. The simulation results 

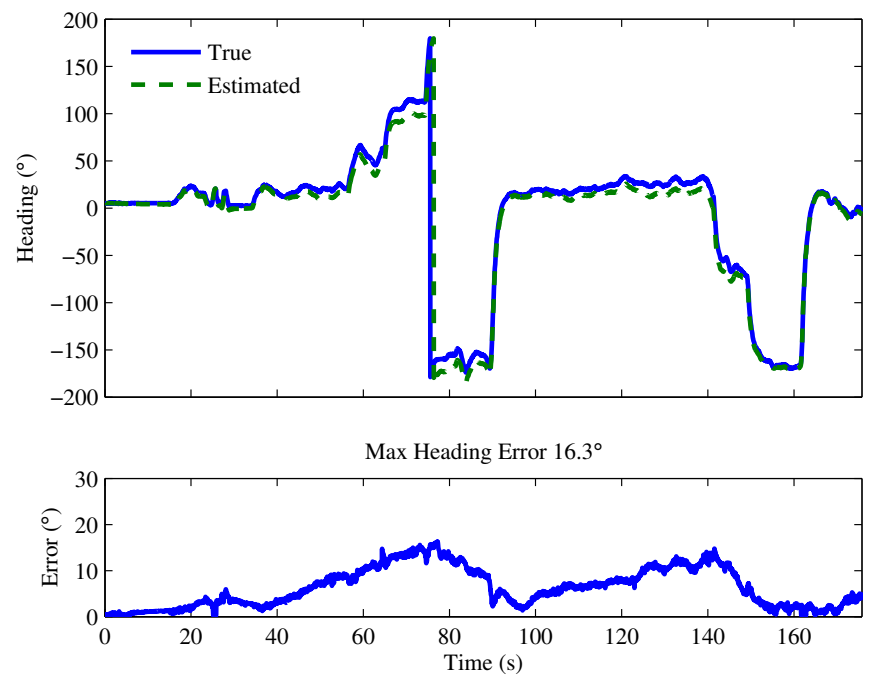

Fig. 2: Test flight using the pure Euler method to correct attitude.
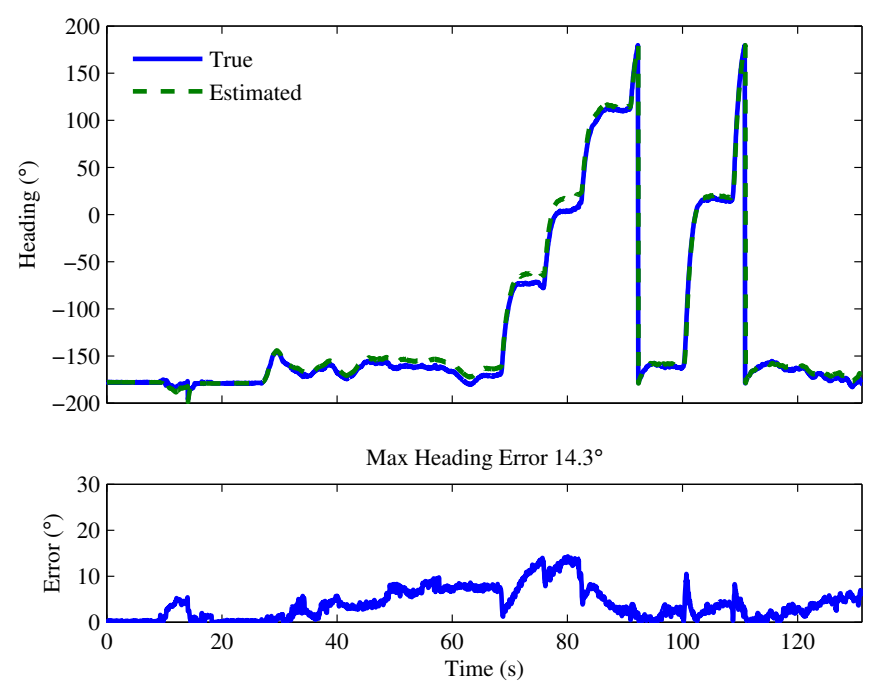

Fig. 3: Test flight using the mixed quaternion Euler method.
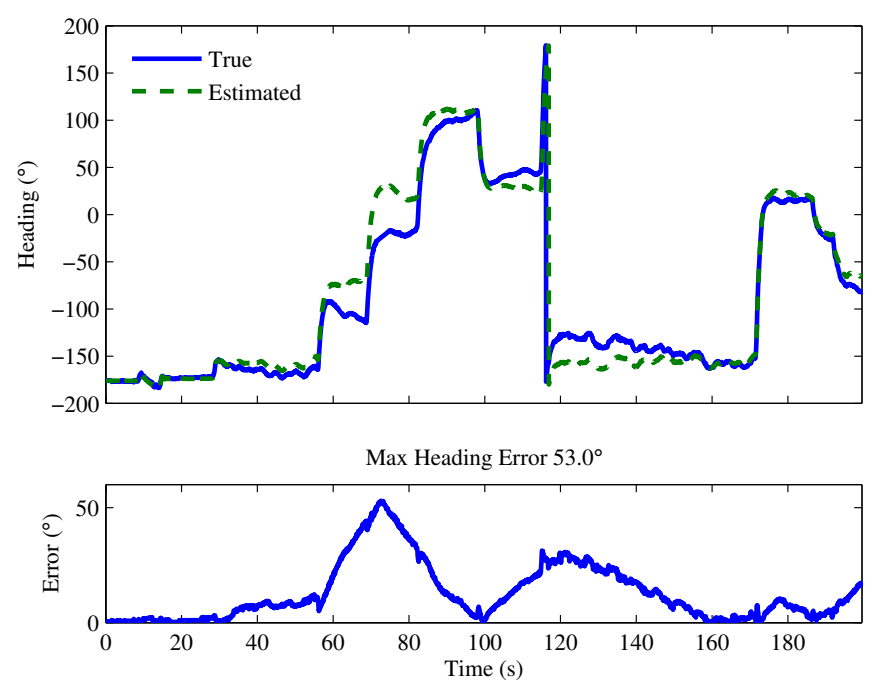

Fig. 4: Test flight using the pure quaternion method.
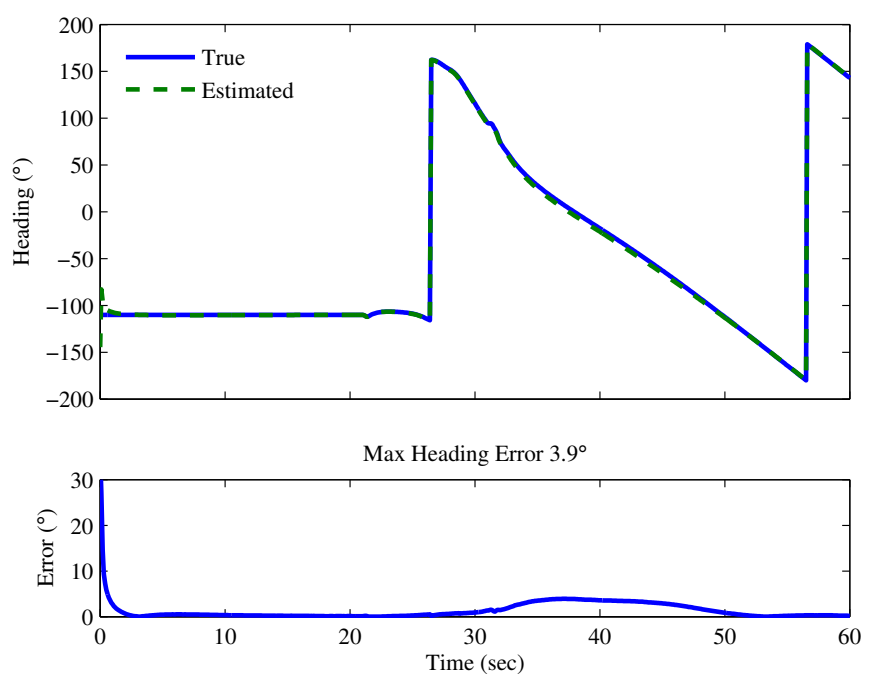

Fig. 5: Simulation of MEKF. The large error at the beginning is from the filter starting from an uninitialized state. The increase in error at $t=30 \mathrm{~s}$ results from a simulated high-g maneuver.

presented in Figure 5 suggest that the MEKF could have a lower error than the other methods tested.

\section{Conclusions}

We have shown how the traditional method of incorporating magnetometer measurements breaks down for tailsitter aircraft. We have also shown multiple ways to work around this with minimal changes to an EKF and presented preliminary hardware results. These results have been limited to hover flight and must be expanded to level flight. Also shown are some fundamental conceptual issues in using an EKF to estimate a quaternion. A simple MEKF that addresses these issues was implemented and is currently under evaluation.

\section{AcKNowledgments}

This research was funded by the Air Force Research Lab through SBIR FA8650-13-C-2322 as a subcontract through the MLB Company.

\section{REFERENCES}

[1] MLB Company. [Online]. Available: http://spyplanes.com/ products-v-bat/

[2] M. Argyle, R. Beard, and S. Morris, "The Vertical Bat Tail-sitter: Dynamic Model and Control Architecture," American Control Conference, pp. 806-811, 2013.

[3] R. W. Beard and T. W. McLain, Small Unmanned Aircraft: Theory and Practice. Princeton, New Jersey: Princeton University Press, 2012.

[4] R. H. Stone, "Control Architecture for a Tail-sitter Unmanned Air Vehicle," 2004 5th Asian Control Conference, vol. 2, pp. 736-744, 2004.

[5] W. F. Phillips, Mechanics of Flight, 2nd ed. Hoboken, New Jersey: John Wiley \& Sons, Inc, 2010.

[6] J. M. Beach, "Development of Tailsitter Hover Estimation and Control," Master's thesis, Brigham Young University, Feb. 2014.

[7] F. L. Markley, "Attitude Error Representations for Kalman Filtering," Journal of Guidance, Control, and Dynamics, vol. 26, no. 2, pp. 311317, Mar. 2003.

[8] J. K. Hall, N. B. Knoebel, and T. W. McLain, "Quaternion Attitude Estimation for Miniature Air Vehicles Using a Multiplicative Extended Kalman Filter," 2008 IEEE/ION Position, Location and Navigation Symposium, pp. 1230-1237, 2008. 